\title{
LINC00511 as a prognostic biomarker for human cancers: a systematic review and meta-analysis
}

Yannick Luther Agbana', Manzama-Esso Abi ${ }^{2}$, Yueli Ni ${ }^{1}$, Guohang Xiong ${ }^{1}$, Jing Chen ${ }^{1}$, Fang Yun ${ }^{1}$, Zihan Yi ${ }^{1}$, Qiao Zhang ${ }^{1}$, Zhe Yang ${ }^{3}$, Yingmin Kuang ${ }^{4}$ and Yuechun Zhu ${ }^{1 *}$

\begin{abstract}
Background: Long intergenic non-coding RNA 00511 (LINC00511) is highly expressed in diverse cancers and has a correlation with poor clinical outcomes for cancer patients. In view of contradictory data among published data, we aim to evaluate the prognostic role of LINC00511 for cancer patients.

Methods: In the present study, a meta-analysis of related studies has been performed to investigate the prognostic significance of LINC00511 in cancer patients. Relevant studies published before December 22, 2019 were systematically searched online in PubMed, EMBASE, Web of Science, and the Cochrane Library databases. The relationship between LINC00511 expression and cancer patients' survival, including overall survival (OS), disease-free survival (DFS)/relapsefree survival (RFS) and progression-free survival (PFS), was evaluated using pooled hazard ratios (HRs) with their corresponding 95\% confidence intervals (Cls). The association between LINC00511 expression and clinicopathological features was assessed using odd ratios (ORs) and their corresponding 95\% Cls.

Results: A total of 14 eligible studies with 1883 patients were enrolled in the present meta-analysis. The results demonstrated that elevated expression of LINC00511 was significantly associated with poor OS (HR $=2.62 ; 95 \% \mathrm{Cl}$ : 2.00-3.45; $p<0.001)$, PFS (HR=1.80; 95\% Cl: 1.29-2.51; $p=0.001)$ and DFS/RFS (HR=2.90; 95\% Cl: 1.04-8.12; $p=0.04)$. Additionally, High LINC00511 expression was associated with large tumor size (OR = 3.10; 95\% Cl: 1.97-4.86; $p<0.00001)$, lymph node metastasis $(\mathrm{OR}=3.11 ; 95 \% \mathrm{Cl}: 2.30-4.21 ; p<0.00001)$, advanced clinical stage $(\mathrm{OR}=3.95$; 95\% Cl: 2.68-5.81; $p<0.00001)$, distant metastasis $(\mathrm{OR}=2.39 ; 95 \% \mathrm{Cl}: 1.16-4.93 ; p=0.02)$, and disease recurrence $(\mathrm{OR}=$ 4.62; 95\% Cl: 2.47-8.65; $p<0.00001$ ). Meanwhile, no correlation was found between LINC00511 expression and age, gender, and histological grade. These findings were consolidated by the results of bioinformatics analysis.
\end{abstract}

Conclusions: Based on our findings, LINC00511 may serve as a novel prognostic biomarker for cancer patients.

Keywords: LINC00511, Prognostic biomarker, Survival, Meta-analysis, TCGA, Cancer

\footnotetext{
* Correspondence: zhuyuechun20091119@163.com

'Department of Biochemistry and Molecular Biology, Kunming Medical University, Kunming 650500, Yunnan Province, China

Full list of author information is available at the end of the article
}

(c) The Author(s). 2020 Open Access This article is licensed under a Creative Commons Attribution 4.0 International License, which permits use, sharing, adaptation, distribution and reproduction in any medium or format, as long as you give appropriate credit to the original author(s) and the source, provide a link to the Creative Commons licence, and indicate if changes were made. The images or other third party material in this article are included in the article's Creative Commons licence, unless indicated otherwise in a credit line to the material. If material is not included in the article's Creative Commons licence and your intended use is not permitted by statutory regulation or exceeds the permitted use, you will need to obtain permission directly from the copyright holder. To view a copy of this licence, visit http://creativecommons.org/licenses/by/4.0/ The Creative Commons Public Domain Dedication waiver (http://creativecommons.org/publicdomain/zero/1.0/) applies to the data made available in this article, unless otherwise stated in a credit line to the data. 


\section{Background}

Long non-coding RNAs (lncRNAs) are referred to as the non-protein coding portion of the transcriptome with length of more than 200 nucleotides. They represent, together with small noncoding RNAs ( $<200$ nucleotides), the biggest part of the transcriptome, since only 1 to $3 \%$ of the transcriptome can code for protein synthesis [1-3]. Long noncoding RNAs are involved in several biological processes including cell proliferation, cell differentiation, cell cycle progression, cell apoptosis and metastasis [410]. Notably, they were identified as oncogenes or tumor suppressors, prognostic and diagnostic biomarkers and as therapeutic targets [11-13]. For instance, Tang and colleagues reviewed the implication of lncRNAs in colorectal cancer progression and figured out their potential clinical applications as novel diagnostic and prognostic biomarkers and therapeutic targets [14].

Long intergenic non-coding RNA 00511 (LINC00511) is a 2265-bp lncRNA mapped to chromosome $17 \mathrm{q} 24.3$ with five exons. Recent studies found that LINC00511 is overexpressed in various type of cancers including breast cancer, ovarian cancer, liver cancer, pancreatic cancer, lung cancer and glioma. LINC00511 is an oncogene which plays a negative regulatory role in cell proliferation, cell cycle progression, apoptosis, invasion, migration, metastasis and chemoresistance [15-23]. Noteworthily, overexpression of LINC00511 was shown to be a predictor for cancer prognosis $[16,18,20,22,24-28]$. However, the results of the studies were not consistent. For instance, the studies of Deng et al. [27], Zhao et al. [20] and Wang et al. [18] showed no correlation between the expression level of LINC00511 and tumor size; in contrast the studies of Sun et al. [22], Yu et al. [25] and Zhang et al. [26] demonstrated an association between LINC00511 expression and tumor size. Similarly, Lu et al. [16] detected significant correlation between LINC00511 overexpression and lymph node metastasis in breast cancer, whereas the study conducted by Zhang et al. [26] provided a contradictory result. In view of these contradictory outcomes, we conducted a systematic review and meta-analysis to evaluate the prognostic role of LINC00511 for cancer patients. Next, we validated our results by using The Cancer Genome Atlas (TCGA) and Genotype-Tissue Expression (GTEx) datasets.

\section{Methods}

\section{Literature search strategies}

The present study was performed according to the Preferred Reporting Items for Systematic Reviews and Meta-Analysis (PRISMA) [29]. Relevant studies published before December 22, 2019 were systematically searched online in PubMed, EMBASE, Web of Science and the Cochrane Library databases. The following search terms were used in different combinations:
(LINC00511 OR "long intergenic noncoding RNA 00511" OR "long intergenic non-protein coding RNA 511") AND (cancer OR tumor OR tumour OR carcinoma OR neoplasm OR adenoma OR sarcoma OR malignancy). The reference lists of the full-text articles were also checked to find relevant studies that pointed out the relation between LINC00511 expression and clinical outcomes. No language restriction was applied.

\section{Inclusion and exclusion criteria}

The eligibility criteria were as follows: (1) the study subjects were patients with any type of cancer; (2) the studies investigated association between LINC00511 expression levels and clinical outcomes in cancer; (3) patients were separated into LINC00511 high expression level and LINC00511 low expression level groups; (4) the LINC00511 expression levels were measured by quantitative method (e.g., realtime reverse transcription polymerase chain reaction (qRTPCR)); and (5) sufficient information and data were provided to calculate a hazard ratios (HRs) or odd ratios (ORs) and their corresponding 95\% confidence intervals (CIs). Exclusion criteria were as follows: (1) duplicate publications; (2) reviews and meta-analyses; (3) studies without patient samples; (4) studies not relevant to cancer, LINC00511 or prognosis.

\section{Publication quality assessment}

The quality of included studies was assessed using the Newcastle-Ottawa Scale (NOS). Three main categories have been considered including selection, comparability and outcome, and the stars rating system has been used. The scores of NOS were ranged from 0 star (lowest score) to 9 stars (highest score). A study with a NOS score higher than 5 was considered as a high-quality study [30,31].

\section{Data extraction}

Two investigators reviewed all eligible studies independently and extracted the following data: the first author's name, year of publication, country of origin, the tumor type, sample size, cut-off value, detection method, HRs and corresponding 95\% CIs, and clinicopathological features. Any controversial issue was resolved by discussion. In case $H R$ value was not provided, Engauge Digitizer version 11.2 (http://markummitchell.github.io/ engauge-digitizer/) was used to extract data from Kaplan-Meier curve. The extracted data were then used to estimate HRs and $95 \%$ CIs by using the HR calculations spreadsheet provided by Tierney et al. [32].

\section{Bioinformatics analysis}

We used GEPIA, a web server for cancer and normal gene expression profiling and interactive analyses, to perform bioinformatics analysis. GEPIA uses the data from both TCGA (The Cancer Genome Atlas) and 
GTEx (Genotype-Tissue Expression) to perform several analyses. The datasets were computed by the UCSC Xena project based on a standard pipeline [33, 34]. Therefore, we directly use GEPIA to perform our analyses. GEPIA used Kaplan-Meier method and log-rank test for the survival analysis and one-way ANOVA for the gene expression analysis. First, the expression levels of LINC00511 in the different cancer types involved in our meta-analysis was analyzed by matching TCGA and GTEx data. Next, overall survival and disease-free survival curves were retrieved.

\section{Statistical analysis}

All statistical analyses were performed with RevMan 5.3 software (Cochrane community, https://community. cochrane.org/help/tools-and-software/revman-5/revman-5-download/) and STATA 15.0. The pooled HRs and their 95\% CIs were used to evaluate the association of LINC00511 expression with the cancer patients' survival. Odd ratios (ORs) analyses were performed to assess the correlation between LINC00511 expression and clinicopathological parameters. The heterogeneity between studies results was examined by Cochran's Q test and Higgins I-squared $\left(\mathrm{I}^{2}\right)$ statistic. In the absence of heterogeneity $\left(p \geq 0.10\right.$ and/or $\left.\mathrm{I}^{2}<50 \%\right)$, the fixed-effect model was used; otherwise the random-effect model was applied [35, 36]. Potential publication bias was evaluated using the funnel plot, Egger's test and Begg's test. Sensitivity analysis was also performed to assess the impact of individual study on the pooled effect [37]. A $p$-value less than 0.05 was considered to be statistically significant.

\section{Results}

Characteristics of included studies

A flowchart of the literature search strategy is shown in Fig. 1. According to the described search strategy, 118

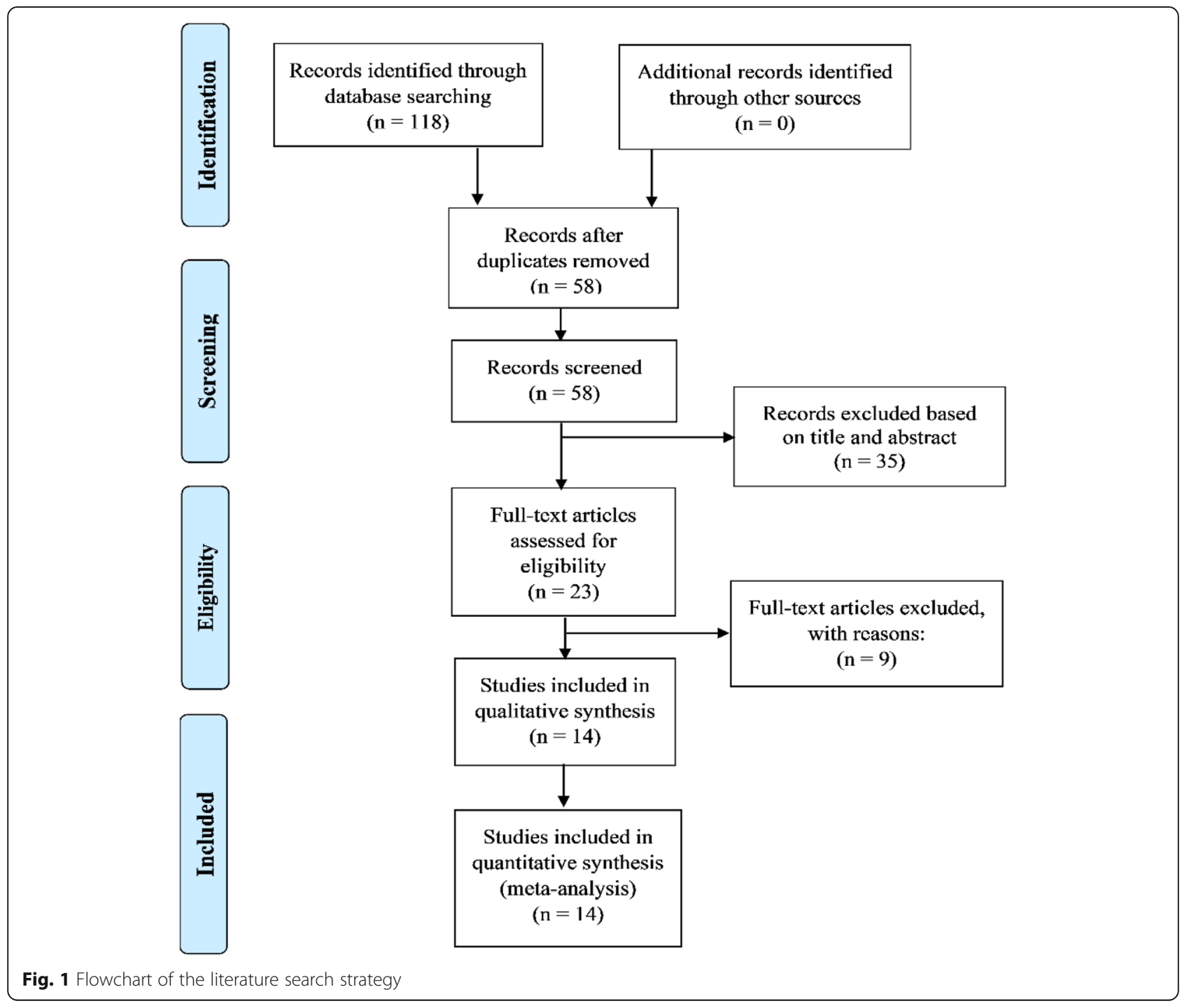


articles were retrieved. Sixty articles were excluded based on duplication criteria. The 58 articles remained, underwent an initial screening. On the basis of title and abstract, 35 articles (non-cancer publications, nonLINC00511 publications, reviews and meta-analyses, retracted article, articles without prognosis and clinical data) were excluded. A total of 23 full-text articles were eventually assessed for eligibility. After reading full-text articles, 9 articles were excluded for reasons: articles without prognosis and clinical data in respect to LINC00511, not available full-text article and nonpatient samples. At the end, 14 articles were used for the quantitative synthesis $[16-22,24-28,38,39]$. The 14 studies involved a total of 1883 patients and all came from China. The sample size of included studies ranged from 36 to 412 patients and the type of cancer included non-small-cell lung cancer, pancreatic cancer, breast cancer, cervical cancer, hepatocellular carcinoma, ovarian cancer, and brain tumors. The articles included in the present study were all written in English and published between 2016 and 2019. The expression level of LINC00511 was mainly determined by qRT-PCR. The cut-off values included mean, median and the P25 value. In our study, the quality of all included studies is high (NOS score $>5$ ). The overall characteristics of included studies are summarized in Table 1.

\section{Association between LINC00511 expression and cancer patients' survival}

Elevated LINC00511 expression was significantly predictive of poor overall survival ( $\mathrm{HR}=2.62$; $95 \% \mathrm{CI}$ : $2.00-$ $3.45 ; p<0.001)$. A high heterogeneity was observed among the studies $\left(\mathrm{P}_{\mathrm{H}}<0.001 ; \mathrm{I}^{2}=85.3 \%\right)$, so the random-effect model was used (Fig. 2a). In addition, high LINC00511 expression has been proven to have an independent prognostic value in four studies by processing the multivariate analysis $[18,20,22,25]$. As previously reported [40], we conducted the meta-analysis of these studies and found that high LINC00511 expression may serve as an independent predictor for poor overall survival prognosis in cancers $(\mathrm{HR}=3.37$; 95\% CI: $1.96-$ 5.79; $p<0.001 ; \mathrm{I}^{2}=82.5 \% ; \mathrm{P}_{\mathrm{H}}=0.001$; Fig. $2 \mathrm{~b}$ ).

The relationship between LINC00511 expression and cancer progression was investigated by combining progression-free survival (PFS) studies. The results, from only two studies with available related data, demonstrated that high LINC00511 expression was predicted to be associated significantly with worse PFS $(\mathrm{HR}=1.80$; 95\% CI: $1.29-2.51 ; p=0.001 ; \mathrm{I}^{2}=0 \% ; \mathrm{P}_{\mathrm{H}}=0.44$; Fig. 2 c). In addition, one study found that high LINC00511 may be associated with worse relapse-free survival $(\mathrm{HR}=$ 2.90; 95\% CI: 1.04-8.12; $p=0.04$ ).

Subgroup meta-analyses based on cancer types (according to NCBI's medical subject headings (MeSH)
[41]) (Fig. 3a), sample size (Fig. 3b), cut-off value (Fig. 3c) and data extraction method (Fig. 3d) were conducted. The results showed that high LINC00511 expression was significantly associated with poor overall survival in lung neoplasms $(\mathrm{HR}=3.86$; 95\% $\mathrm{CI}$ : $1.74-$ 8.54; $\left.p=0.001 ; \mathrm{I}^{2}=80.5 \% ; \mathrm{P}_{\mathrm{H}}=0.02\right)$, digestive system neoplasms $\left(\mathrm{HR}=3.06\right.$; 95\% CI: $2.76-3.40 ; p<0.001 ; \mathrm{I}^{2}=$ $\left.0 \% ; \mathrm{P}_{\mathrm{H}}=0.45\right)$, breast neoplasms $(\mathrm{HR}=2.20 ; 95 \% \mathrm{CI}$ : $\left.1.50-3.23 ; p<0.001 ; \mathrm{I}^{2}=0 \% ; \mathrm{P}_{\mathrm{H}}=0.99\right)$ and urothelial neoplasms (HR $=3.15$; 95\% CI: 2.60-3.80; $p<0.001$; $\mathrm{I}^{2}=$ $0 \% ; \mathrm{P}_{\mathrm{H}}=0.80$ ). Regarding the brain neoplasms subgroup, despite the relatively high $\mathrm{HR}$, the relationship cannot be considered robust because the $p$-value is higher than $0.05\left(\mathrm{HR}=1.50 ; 95 \% \mathrm{CI}: 0.87-2.60 ; p=0.15 ; \mathrm{I}^{2}=68.6 \%\right.$; $\left.\mathrm{P}_{\mathrm{H}}=0.07\right)$. In respect to sample size subgroups, there was significant correlation between high LINC00511 expression and poor overall survival prognosis of cancer patients in both large sample size $(n \geq 95)(\mathrm{HR}=2.65$; 95\% CI: $\left.1.81-3.89 ; p<0.001 ; \mathrm{I}^{2}=92.9 \% ; \mathrm{P}_{\mathrm{H}}<0.001\right)$ and small sample size $(n<95)(\mathrm{HR}=2.63 ; 95 \%$ CI: $2.02-3.43$; $\left.p<0.001 ; \mathrm{I}^{2}=0 \%, \mathrm{P}_{\mathrm{H}}=0.79\right)$. Concerning the cut-off value subgroups analysis, whether the median was used as cut-off value $(\mathrm{HR}=2.86$; 95\% CI: $2.43-3.38 ; p<0.001$; $\left.\mathrm{I}^{2}=0 \% ; \mathrm{P}_{\mathrm{H}}=0.87\right)$ or the mean as cut-off value $(\mathrm{HR}=$ 2.24; 95\% CI: $\left.1.38-3.64 ; p=0.001 ; \mathrm{I}^{2}=0 \%, \mathrm{P}_{\mathrm{H}}=0.99\right)$ or other cut-off values were used (HR $=2.80$; $95 \%$ CI: 1.60 4.90; $\left.p<0.001 ; \mathrm{I}^{2}=95.2 \% ; \mathrm{P}_{\mathrm{H}}<0.001\right)$, high LINC00511 expression correlated with poor overall survival in cancer patients. Similarly, the subgroup meta-analysis based on the data extraction method showed that whether the data were extracted directly from the literature $(\mathrm{HR}=$ 3.21; 95\% CI: 2.73-3.76; $p<0.001 ; \mathrm{I}^{2}=37.4 \% ; \mathrm{P}_{\mathrm{H}}=0.14$ ) or indirectly $(\mathrm{HR}=1.80 ; 95 \% \mathrm{CI}: 1.30-2.47 ; p<0.001$; $\mathrm{I}^{2}=41.6 \% ; \mathrm{P}_{\mathrm{H}}=0.11$ ), high LINC00511 expression was significantly associated with poor overall survival in cancer patients. Of note, the heterogeneity is likely to come from diverse sources.

\section{Association between LINC00511 expression and clinicopathological features}

The meta-analysis of the correlation between LINC00511 expression and clinicopathological parameters are summarized in Table 2. The pooled odds ratio (OR) values revealed significant association between high LINC00511 expression and large tumor size $(\mathrm{OR}=3.10$; 95\% CI: $\left.1.97-4.86 ; \quad p<0.00001 ; \mathrm{I}^{2}=50 \% ; \quad \mathrm{P}_{\mathrm{H}}=0.03\right)$, lymph node metastasis $(\mathrm{OR}=3.11$; $95 \% \mathrm{CI}$ : $2.30-4.21$; $\left.p<0.00001 ; \mathrm{I}^{2}=42 \% ; \mathrm{P}_{\mathrm{H}}=0.08\right)$, advanced clinical stage $\left(\mathrm{OR}=3.95 ; 95 \%\right.$ CI: 2.68-5.81; $p<0.00001 ; \mathrm{I}^{2}=0 \% ; \mathrm{P}_{\mathrm{H}}=$ $0.48)$, distant metastasis $(\mathrm{OR}=2.39$; $95 \%$ CI: $1.16-4.93$; $\left.p=0.02 ; \quad \mathrm{I}^{2}=21 \% ; \mathrm{P}_{\mathrm{H}}=0.28\right)$, and disease recurrence $\left(\mathrm{OR}=4.62 ; 95 \%\right.$ CI: $2.47-8.65 ; p<0.00001 ; \mathrm{I}^{2}=0 \% ; \mathrm{P}_{\mathrm{H}}=$ 0.66). Meanwhile, the results showed no statistically 


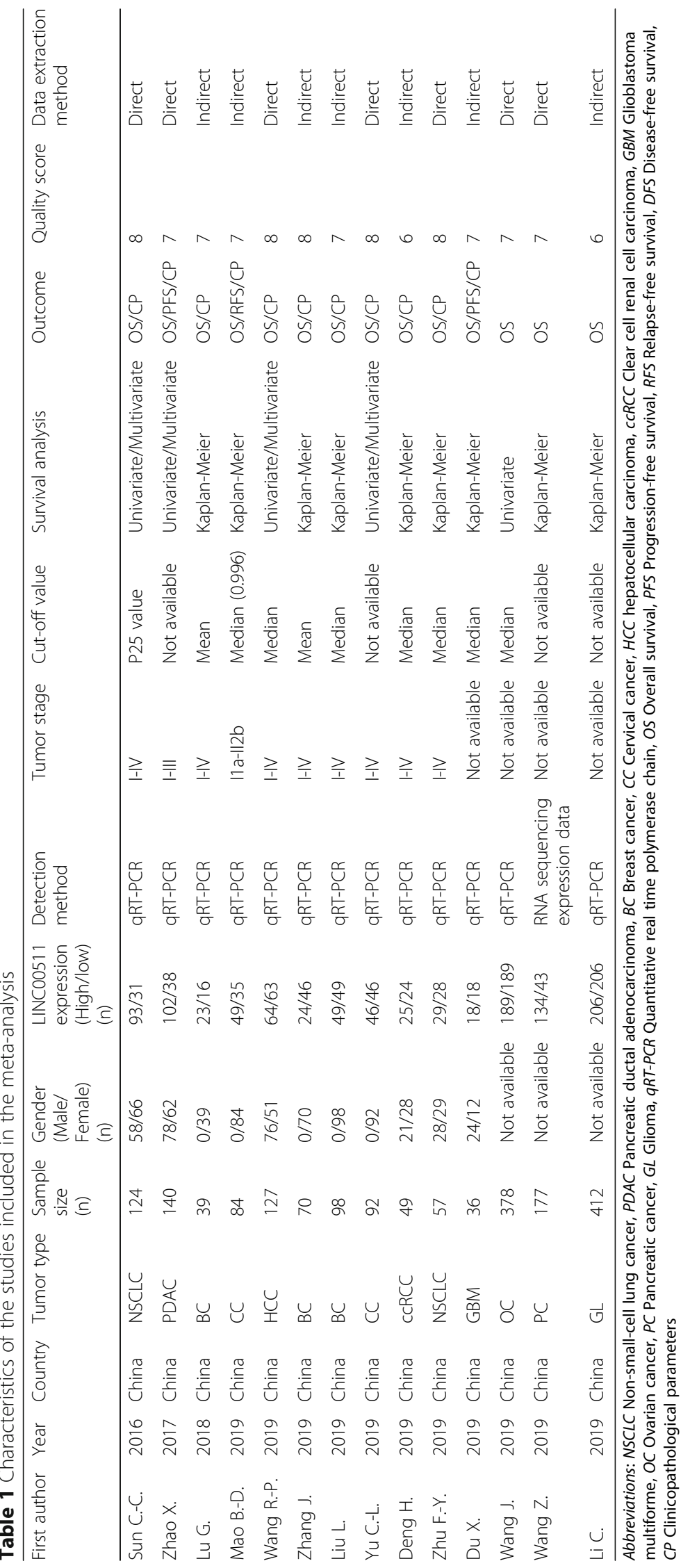




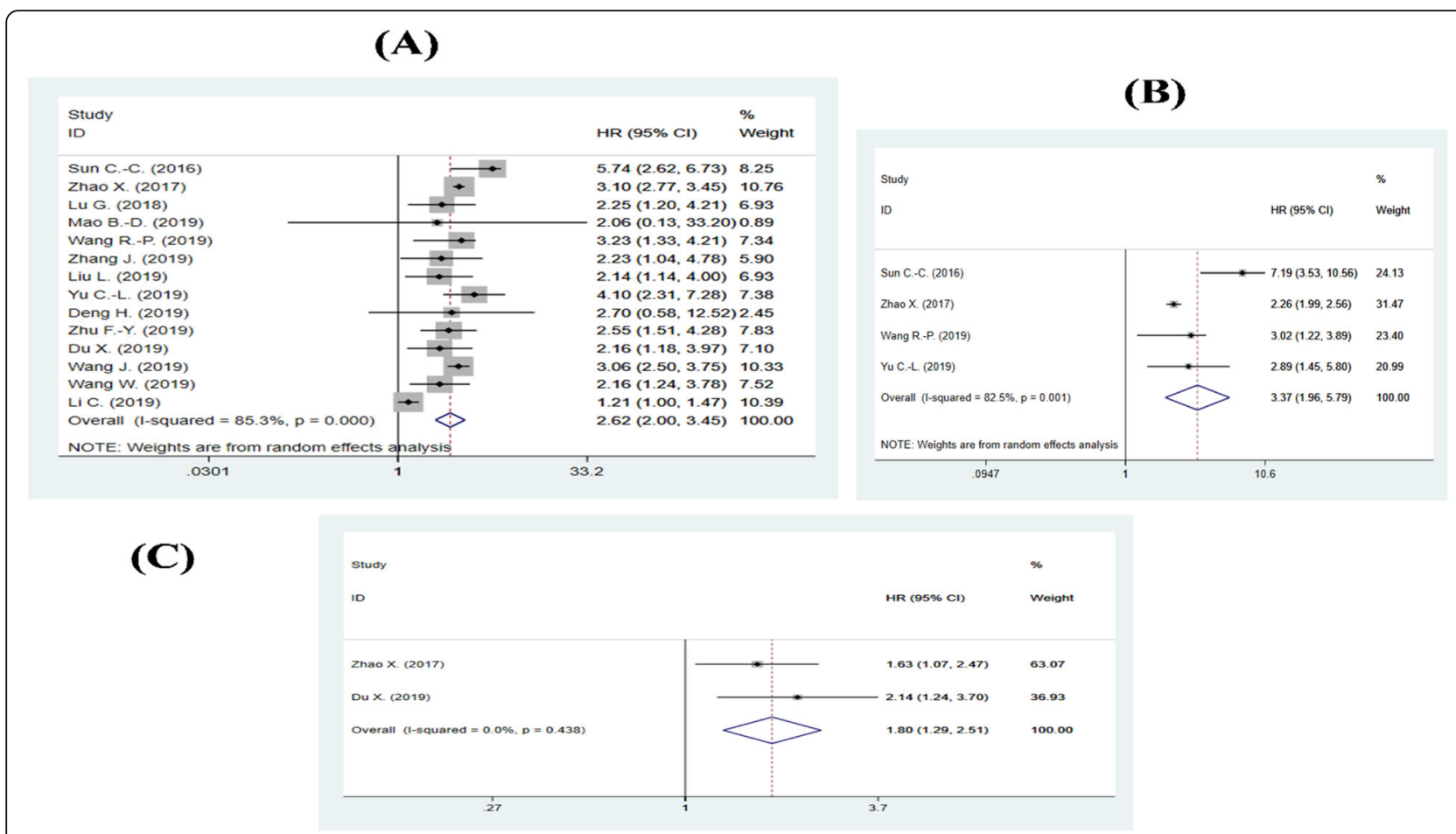

Fig. 2 Forest plots of combined analyses on the association of survival with LINC00511 expression. a Forest plot of OS analysis, $\mathbf{b}$ Forest plot of meta-analysis of the independent predictive value of LINC00511 for OS, and c forest plot of PFS analysis

(A)

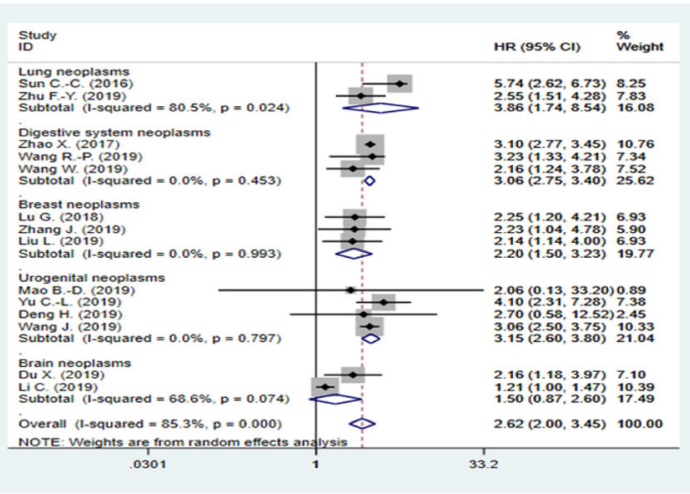

(C)

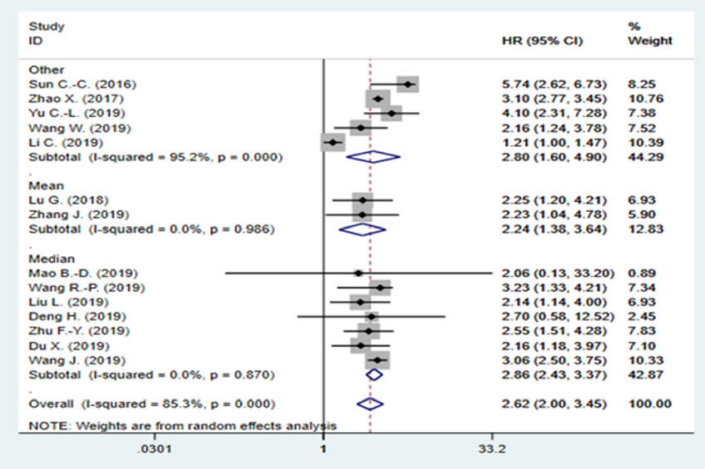

(B)

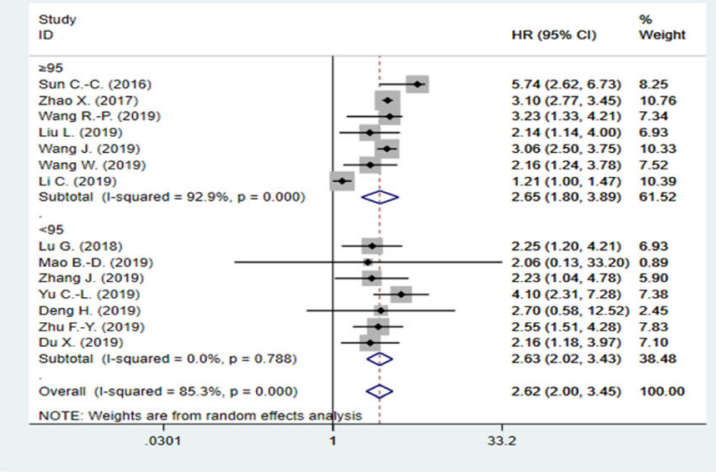

(D)

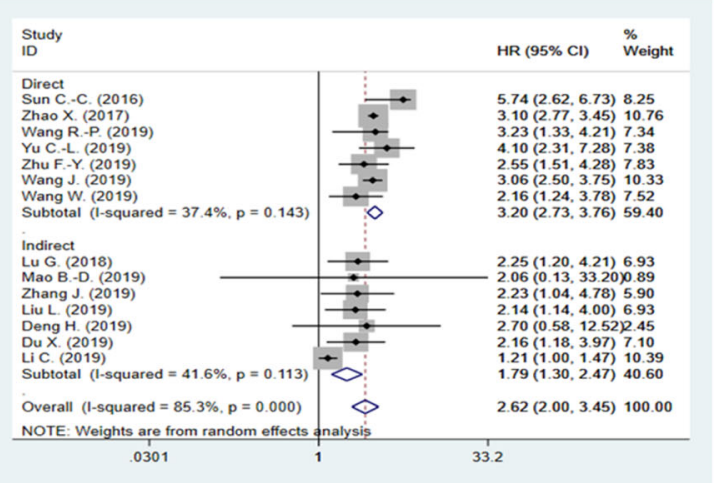

Fig. 3 Forest plots of the association between LINC00511 expression and overall survival in subgroups based on a cancer types; b sample size; $\mathbf{c}$ different cut-off value and $\mathbf{d}$ different extraction method 
Table 2 Meta-analyses of the correlation between LINC00511 expression and clinicopathological features

\begin{tabular}{|c|c|c|c|c|c|c|c|}
\hline \multirow[t]{2}{*}{ Variables } & \multirow{2}{*}{$\begin{array}{l}\text { Number } \\
\text { of studies }\end{array}$} & \multirow{2}{*}{$\begin{array}{l}\text { Number } \\
\text { of patients }\end{array}$} & \multirow[t]{2}{*}{ Model } & \multicolumn{2}{|l|}{ OR } & \multicolumn{2}{|c|}{ Heterogeneity } \\
\hline & & & & $95 \% \mathrm{Cl}$ & $p$-value & $\overline{P^{2}(\%)}$ & $\mathrm{P}_{\mathrm{H}}$ \\
\hline Gender (Male vs. Female) & 6 & 498 & Random & $0.98(0.53-1.85)$ & 0.96 & 58 & 0.04 \\
\hline \multicolumn{8}{|l|}{ Age } \\
\hline$\geq 45$ vs. $<45$ & 1 & 84 & - & $0.66(0.28-1.59)$ & 0.36 & - & - \\
\hline$\geq 50$ vs. $<50$ & 6 & 462 & Fixed & $0.69(0.47-1.01)$ & 0.06 & 0 & 0.98 \\
\hline$\geq 60$ vs. $<60$ & 1 & 140 & - & $0.72(0.34-1.52)$ & 0.39 & - & - \\
\hline$\geq 65$ vs. $<65$ & 2 & 173 & Fixed & $0.59(0.29-1.20)$ & 0.15 & 0 & 0.89 \\
\hline Histological grade (poor vs. well and moderate) & 8 & 725 & Fixed & $1.34(0.97-1.85)$ & 0.07 & 0 & 0.75 \\
\hline $\begin{array}{l}\text { Histological tumor type (adenocarcinoma vs. } \\
\text { squamous cell carcinoma) }\end{array}$ & 3 & 300 & Random & $2.80(0.58-13.59)$ & 0.20 & 71 & 0.03 \\
\hline Tumor size (large vs. small) & 11 & 916 & Random & $3.10(1.97-4.86)$ & $<0.00001$ & 50 & 0.03 \\
\hline Lymph node metastasis (positive vs. negative) & 10 & 880 & Fixed & $3.11(2.30-4.21)$ & $<0.00001$ & 42 & 0.08 \\
\hline Clinical stage (late vs. early) & 8 & 643 & Fixed & $3.95(2.68-5.81)$ & $<0.00001$ & 0 & 0.48 \\
\hline Distant metastasis (Yes vs. No) & 3 & 215 & Fixed & $2.39(1.16-4.93)$ & 0.02 & 21 & 0.28 \\
\hline Recurrence (Yes vs. No) & 3 & 274 & Fixed & $4.62(2.47-8.65)$ & $<0.00001$ & 0 & 0.66 \\
\hline
\end{tabular}

Abbreviations: $O R$ Odd ratio, $P_{H} P$-value of heterogeneity

significant correlation between LINC00511 expression and age, gender, and histological grade.

\section{Publication bias}

Funnel plot analysis, Egger's test and Begg's test were performed to evaluate potential publication bias. There was no obvious asymmetry on the funnel plot of OS (Fig. 4) and the tests of publication bias (Begg's test: $p=0.161$; Egger's test: $p=0.630$ ) indicated that no publication bias existed in studies reported OS.

\section{Sensitivity analysis}

The sensitivity analysis was performed by omitting each individual study to test the stability of the pooled result of the association between LINC00511 expression and OS. As shown on Fig. 5, when "Li C. (2019)" was omitted, the pooled result oscillated. Subsequently, we evaluated the pooled HR after removing "Li C. (2019)" and found that elevated LINC00511 expression still predicted worse OS (HR $=3.06 ; 95 \%$ CI: $2.80-3.33 ; p<0.001 ; \mathrm{I}^{2}=$ 14.8\%; $\left.P_{H}=0.30\right)$. Therefore, the influential study didn't alter the significance of the pooled result, sustaining the reliability of our pooled result.

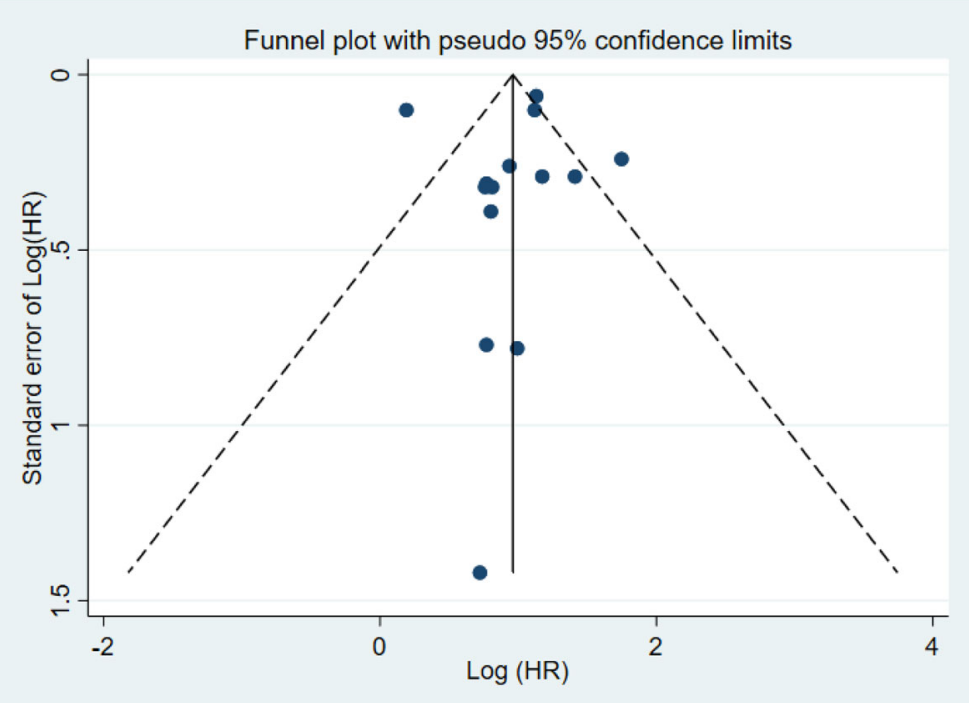

Fig. 4 Funnel plot of publication bias based on Overall survival (OS) 


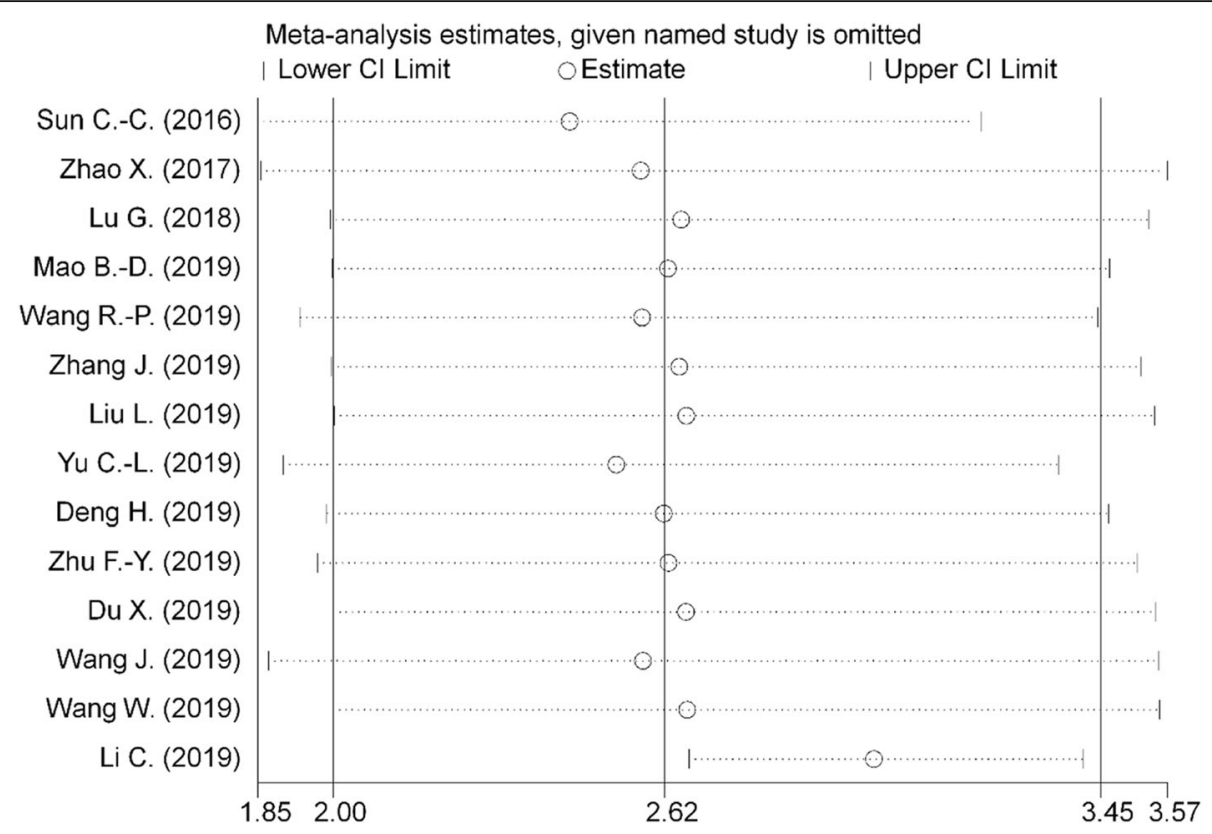

Fig. 5 Sensitivity analysis of pooled HR for overall survival

\section{Datasets analyses by GEPIA}

To strengthen the results of our meta-analysis, we used GEPIA web server which contain a larger amount of sample size and performed bioinformatics analysis. The expression level of LINC00511 was analyzed in eleven different type of cancers (cancer types included in the meta-analysis). As shown on the Fig. 6, LINC00511 was highly expressed in almost all the related cancers, including lung adenocarcinoma (LUAD), lung squamous cell carcinoma (LUSC), pancreatic adenocarcinoma (PAAD), breast invasive carcinoma (BRCA), cervical squamous cell carcinoma (CESC), liver hepatocellular carcinoma (LIHC), kidney renal clear cell carcinoma (KIRC), kidney renal papillary cell carcinoma (KIRP), kidney chromophobe $(\mathrm{KICH})$, glioblastoma multiforme (GBM), and brain lower grade glioma (LGG) $\left(\log _{2} \mathrm{FC}\right.$ value $>1$ and $p$-value $<0.01)$. The Fig. 7 shows the Kaplan-Meier curves of the overall survival (Fig. 7a) and disease-free survival (Fig. 7b) analyses. A total of 4410 patients were divided into LINC00511 low expression and high expression groups based on the median LINC00511 expression. The patients in high expression group showed a worst overall survival $(\mathrm{HR}=2.00$; $\log$ rank $p$-value $<0.001)$ and disease-free survival $(\mathrm{HR}=1.8$; log-rank $p$-value $<0.001)$ than those in the low expression group. Furthermore, we explored the possibility for any association of the LINC00511 expression to overall cancer survival in other cancer types that are not involved in the meta-analysis. It was found that LINC00511 overexpression was significantly associated with worse OS in adrenocortical carcinoma (ACC)
$(\mathrm{HR}=4.5 ; \quad \log$-rank $p$-value $=0.00047 ; \quad$ Fig. $7 \mathrm{c})$, and thymoma $(\mathrm{THYM})(\mathrm{HR}=7.00$; log-rank $p$-value $=0.035$; Fig. 7d). These results confirmed that LINC00511 is overexpressed in different types of cancers and this is correlated with poor survival. Of note, the GEPIA web server was accessed on December 28, 2019.

\section{Discussion}

LINC00511, a newly identified lncRNA, has been reported to be upregulated and to have an oncogenic function in diverse cancers including lung cancer, breast cancer, pancreatic cancer, cervical cancer, liver cancer, ovarian cancer and glioma. Its underlying mechanisms of action include promotion of proliferation, tumorigenesis, cell cycle progression, invasion, migration, metastasis and chemoresistance and inhibition of apoptosis [1622, 24]. More importantly, upregulated LINC00511 has been associated with prognosis, suggesting that it can represent a biomarker for prognosis in cancer patients. However, there is still discrepancy regarding the relationship between LINC00511 expression and clinical outcomes. In the present study, a first-time, comprehensive meta-analysis on the prognostic value of LINC00511 in diverse human cancers is presented. Collectively, 14 eligible studies were systematically included.

The results obtained from this meta-analysis have shown that upregulated LINC00511 is strongly predictive of poor overall survival of cancer patients. Moreover, the combination of multivariate analysis of four relevant studies showed that LINC00511 may serve as an independent predictor of poor overall survival for cancer 

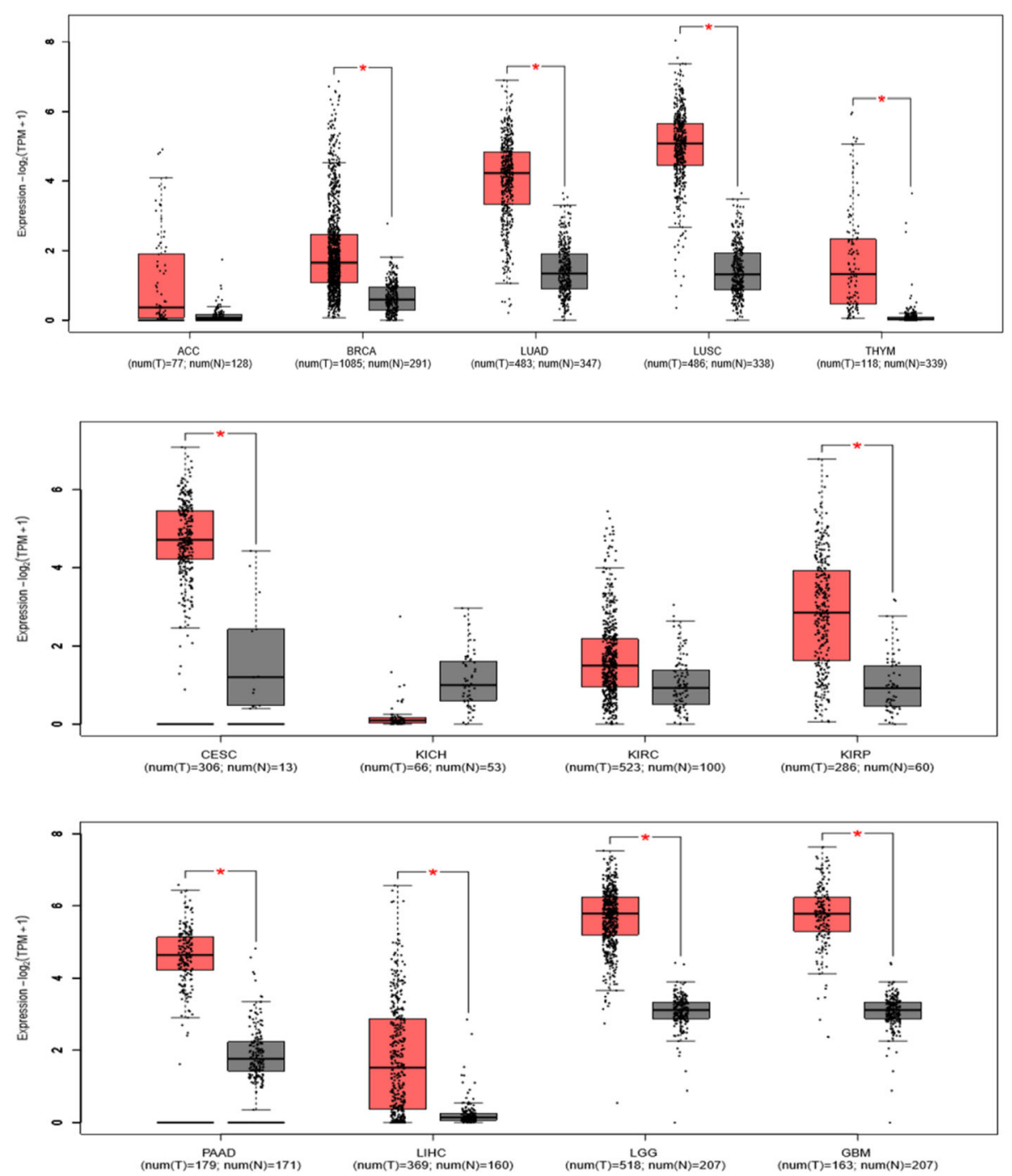

Fig. 6 Validation of the expression levels of LINC00511 in TCGA normal and GTEx datasets: "**" indicates $\log _{2}$ FC value $>1$ and $p$-value $<0.01$. TCGA, The Cancer Genome Atlas; GTEx, Genotype-Tissue Expression; ACC, adrenocortical carcinoma; THYM, thymoma; LGG, brain lower grade glioma; GBM, glioblastoma multiforme; LUAD, lung adenocarcinoma; LUSC, lung squamous cell carcinoma; PAAD, pancreatic adenocarcinoma; BRCA, breast invasive carcinoma; CESC, cervical squamous cell carcinoma; LIHC, liver hepatocellular carcinoma; KIRC, Kidney renal clear cell carcinoma; KIRP, Kidney renal papillary cell carcinoma; KICH, Kidney Chromophobe; TPM, transcripts per million; T, tumor; N, normal

patients. The subgroup analysis revealed that elevated LINC00511 expression correlated with poor overall survival independently to the tumor type, sample size, cutoff value and the data extraction method. Nevertheless, some subgroup analysis results should be taken with caution, due to the small number of studies included. Subsequently, these findings were corroborated by the results of TCGA analysis. Additionally, it was found that patients with elevated LINC00511 expression were more prone to worse clinicopathological features including larger tumor size, advanced clinical tumor stage, lymph node metastasis, distant metastasis and disease recurrence. Besides, high LINC00511 expression was found to be associated with poor disease-free survival and progression-free survival.

Taken together, the above findings lead to the suggestion that LINC00511 could serve as a potential biomarker and functional regulator in human cancers. Molecular mechanisms investigations highlighted that LINC00511 exerts its oncogenic function mainly by modulating microRNAs functions [42-44]. For instance, via its competing endogenous RNA activity on hsa-miR29b-3p, LINC00511 induced the expression of VEGFA leading functionally to pancreatic ductal adenocarcinoma progression [20]. Similarly, Lu et al. [16] found that LINC00511 targeted the miR-185-3p/E2F1/Nanog axis 

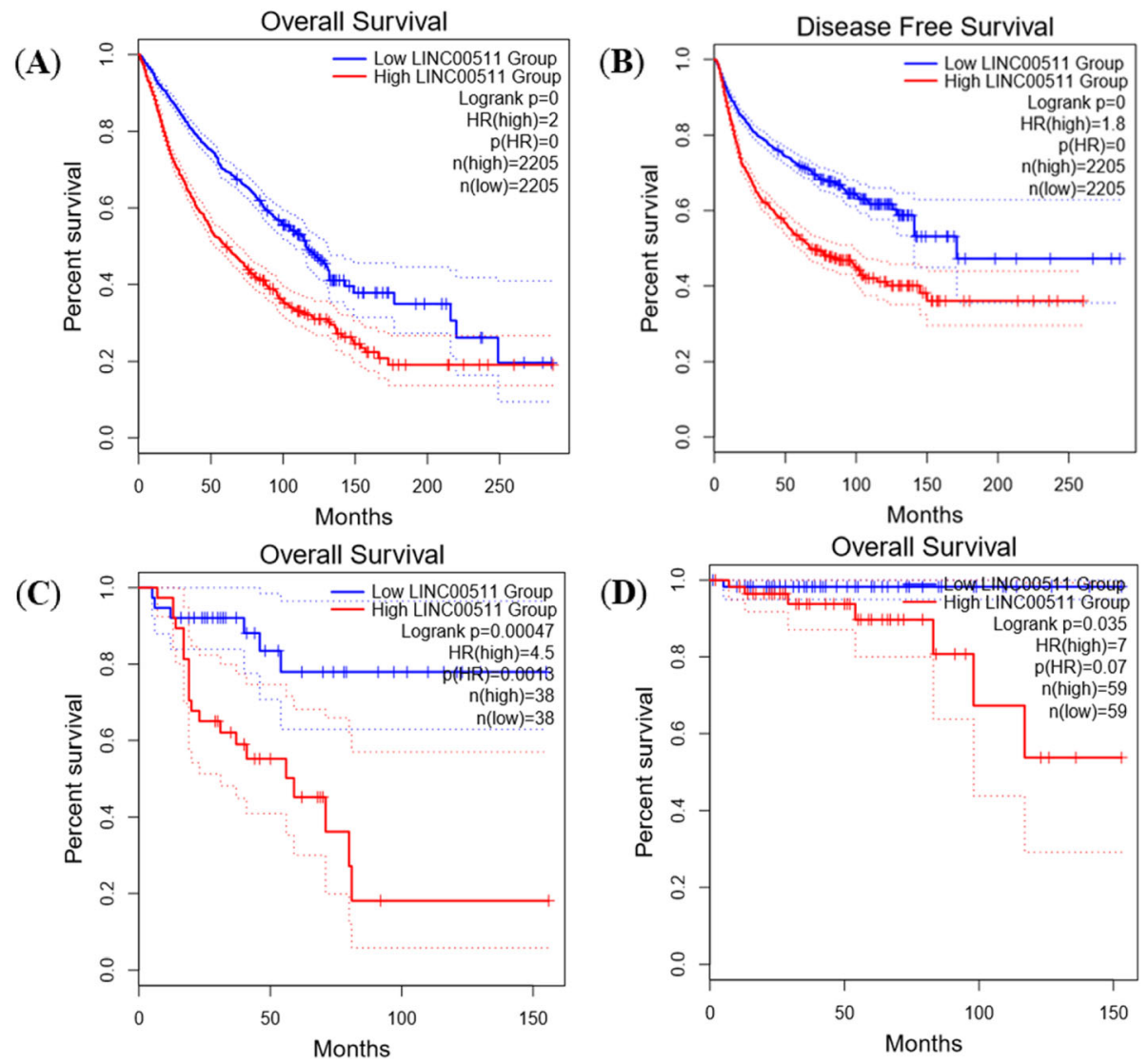

Fig. 7 Kaplan-Meier plots depicting the prognostic potential of LINC00511 for cancer patients' survival. a Overall survival plot. b Disease-free survival. c Overall survival plot for adrenocortical carcinoma (ACC). d Overall survival plot for thymoma (THYM)

in order to promote breast cancer tumorigenesis and stemness. In a recent study, LINC00511 was also found to foster the process of gastric cancer by targeting miR625-5p/NFIX axis [43]. These mechanistic studies revealed that LINC00511 could act at transcriptional and post-transcriptional level. Moreover, LINC00511 was found to interact with a variety of signaling pathways including JAK2/STAT3 [45], Wnt/B-catenin [46], and PTEN/AKT/FOXO1 [47], in the pathogenesis of cancers.

LINC00511 has been demonstrated to be a poor predictor for both cancer recurrence and progression. These analogous outcomes imply that there might be similar LINC0511-dependent mechanisms underlying these two events. In particular, LINC00511 has been shown to induce radio-resistance in breast cancer resulting in recurrence and progression by regulating STXBP4 expression via miR-185 [28]. Similarly, the silencing of LINC00511 in cervical cancer cells enhanced cancer drug paclitaxel's sensitivity, suppressed cell viability, cell proliferation, migration and invasion, and promoted apoptosis, thereby preventing progression and recurrence [24]. To achieve those functions, LINC00511 modulated the expression of related proteins, namely Bcl-2, Bax, cleaved caspase-3, metalloproteinases 2 and 9, multidrug resistance protein 1 (MRP1) and Pglycoprotein. The resistance to paclitaxel caused by LINC00511 was also found in breast cancer and is mediated via regulating miR-29c/CDK6 axis [23]. Du et al. found, in glioblastoma multiforme (GBM), that high LINC00511 expression was correlated with recurrence, and ectopic LINC00511 enhanced GBM cells proliferation, EMT, migration and invasion by sponging miR524-5p to indirectly regulate YB1/ZEB1 [39].

A number of limitations should be addressed when considering the findings of the present study. Firstly, all the studies included in the meta-analysis have been conducted in China, narrowing the representativeness of the results. Secondly, there is a rather limited number of studies available on major cancers, such as lung neoplasms and brain neoplasms. Therefore, the results on subgroup analysis based on cancer types should be taken with cautions. Thus, more clinical studies would be necessary to better assess the relationship between LINC00511 expression and cancer patients' clinical outcomes. 


\section{Conclusions}

In summary, this study demonstrates that the overexpression of LINC00511 can strongly predict a poor survival in cancer patients and that it is associated to large tumor size, lymph node metastasis, advanced clinical stage, distant metastasis and disease recurrence. LINC00511 can thus serve as prognostic biomarker for cancer patients. Nevertheless, further large-scale and high-quality studies from different ethnic background are needed to confirm the present analysis.

\section{Abbreviations}

ACC: Adrenocortical carcinoma; BC: Breast cancer; BRCA: Breast invasive carcinoma; CC: Cervical cancer; cCRCC: Clear cell renal cell carcinoma; CESC: Cervical squamous cell carcinoma; CP: Clinicopathological parameters; DFS: Disease-free survival; GBM: Glioblastoma multiforme; GL: Glioma; GTEx: Genotype-Tissue Expression; HCC: Hepatocellular carcinoma; $\mathrm{KICH}$ : Kidney chromophobe; KIRC: Kidney renal clear cell carcinoma; KIRP: Kidney renal papillary cell carcinoma; LGG: Brain lower grade glioma; LINC00511: Long intergenic non-coding RNA 00511; LIHC: Liver hepatocellular carcinoma; LUAD: Lung adenocarcinoma; LUSC: Lung squamous cell carcinoma; N: Normal; NOS: Newcastle-Ottawa Scale; NSCL C: Non-small-cell lung cancer; OC: Ovarian cancer; OS: Overall survival; PAAD: Pancreatic adenocarcinoma; PC: Pancreatic cancer; PDAC: Pancreatic ductal adenocarcinoma; PFS: Progression-free survival; qRT-PCR: Quantitative real time polymerase chain; RFS: Relapse-free survival; T: Tumor; TCGA: The Cancer Genome Atlas; THYM: Thymoma; TPM: Transcripts per million

\section{Acknowledgments}

Not applicable.

\section{Authors' contributions}

Conceptualization, $Y L A$ and $Y Z$; Formal analysis, $Y L A, M A$ and $Y N$; Investigation, YLA, MA, YN, GX, JC and ZY; Methodology, YLA; Resources, QZ, $Z Y$ and $Y Z$; Supervision, $Y K$ and $Y Z$; Writing - original draft, $Y L A, M A$ and $Q Z$; Writing - review \& editing, FY, ZY and YZ. All authors read and approved the final manuscript.

\section{Funding}

This work was supported by the National Natural Science Foundation of China (No. 31660246, 31960200,81960462,31960145,81460421,81760455, $81560037,91660135)$ and Yunnan Province, Kunming Medical University joint Foundation for Applied Basic Research (No. 2017FE468(- 003), 2018FE468(001), 2017FE468(-132)). The funding bodies played no role in the design of the study and collection, analysis, and interpretation of data and in writing the manuscript.

\section{Availability of data and materials}

All data are included in this article.

Ethics approval and consent to participate

Not applicable.

\section{Consent for publication}

Not applicable.

\section{Competing interests}

The authors declare that they have no competing interests.

\section{Author details}

${ }^{1}$ Department of Biochemistry and Molecular Biology, Kunming Medical University, Kunming 650500, Yunnan Province, China. ${ }^{2}$ Department of Microbiology and Immunology, Kunming Medical University, Kunming 650500, Yunnan Province, China. ${ }^{3}$ Department of Pathology, The First Affiliated Hospital of Kunming Medical University, Wuhua District, Kunming 650032, Yunnan Province, China. ${ }^{4}$ Department of Organ Transplantation, The First Affiliated Hospital of Kunming Medical University, Wuhua District, Kunming 650032, Yunnan Province, China.
Received: 12 March 2020 Accepted: 16 July 2020

Published online: 22 July 2020

\section{References}

1. Frith MC, Bailey TL, Kasukawa T, Mignone F, Kummerfeld SK, Madera M, Sunkara S, Furuno M, Bult CJ, Quackenbush J, et al. Discrimination of nonprotein-coding transcripts from protein-coding mRNA. RNA Biol. 2006;3:40-8.

2. Richtig G, Ehall B, Richtig E, Aigelsreiter A, Gutschner T, Pichler M. Function and clinical implications of long non-coding RNAs in melanoma. Int J Mol Sci. 2017;18 Available from: https://www.ncbi.nlm.nih.gov/pmc/articles/ PMC5412301/. Cited 2019 Jun 10.

3. An integrated encyclopedia of DNA elements in the human genome. Nature. 2012;489:57-74 Available from: https://www.ncbi.nlm.nih.gov/pmc/ articles/PMC3439153/. Cited 2019 Jun 10.

4. Hu X, Sood AK, Dang CV, Zhang L. The role of long noncoding RNAs in cancer: the dark matter matters. Curr Opin Genet Dev. 2018;48:8-15.

5. Chen L, Yang H, Xiao Y, Tang X, Li Y, Han Q, Fu J, Yang Y, Zhu Y. Lentiviralmediated overexpression of long non-coding RNA GAS5 reduces invasion by mediating MMP2 expression and activity in human melanoma cells. Int J Oncol. 2016:48:1509-18.

6. Chen L, Yang H, Xiao Y, Tang X, Li Y, Han Q, Fu J, Yang Y, Zhu Y. LncRNA GAS5 is a critical regulator of metastasis phenotype of melanoma cells and inhibits tumor growth in vivo. OncoTargets Ther. 2016;9:4075-87.

7. Chen L, Yang H, Yi Z, Jiang L, Li Y, Han Q, Yang Y, Zhang Q, Yang Z, Kuang $Y$, et al. LncRNA GAS5 regulates redox balance and dysregulates the cell cycle and apoptosis in malignant melanoma cells. J Cancer Res Clin Oncol. 2019;145:637-52.

8. Roberts TC, Morris KV, Wood MJA. The role of long non-coding RNAs in neurodevelopment, brain function and neurological disease. Philos Trans $R$ Soc Lond Ser B Biol Sci. 2014;369(1652):20130507.

9. Shi C, Zhang L, Qin C. Long non-coding RNAs in brain development, synaptic biology, and Alzheimer's disease. Brain Res Bull. 2017;132:160-9.

10. Li Y, Egranov SD, Yang L, Lin C. Molecular mechanisms of long noncoding RNAs-mediated cancer metastasis. Genes Chromosomes Cancer. 2019;58: 200-7.

11. Pecero ML, Salvador-Bofill J, Molina-Pinelo S. Long non-coding RNAs as monitoring tools and therapeutic targets in breast cancer. Cell Oncol Dordr. 2019;42:1-12.

12. Li M, Wang Y, Cheng L, Niu W, Zhao G, Raju JK, Huo J, Wu B, Yin B, Song Y, et al. Long non-coding RNAs in renal cell carcinoma: a systematic review and clinical implications. Oncotarget. 2017:8:48424-35.

13. Lorenzi L, Avila Cobos F, Decock A, Everaert C, Helsmoortel H, Lefever S, Verboom K, Volders P-J, Speleman F, Vandesompele J, et al. Long noncoding RNA expression profiling in cancer: challenges and opportunities. Genes Chromosomes Cancer. 2019;58:191-9.

14. Tang X, Qiao X, Chen C, Liu Y, Zhu J, Liu J. Regulation mechanism of long noncoding RNAs in colon cancer development and progression. Yonsei Med J. 2019:60:319-25.

15. Yang F, Lyu S, Dong S, Liu Y, Zhang X, Wang O. Expression profile analysis of long noncoding RNA in HER-2-enriched subtype breast cancer by nextgeneration sequencing and bioinformatics. OncoTargets Ther. 2016;9:76172 Available from: http://www.embase.com/search/results?subaction= viewrecord\&from=export\&id $=L 608355680$.

16. Lu G, Li Y, Ma Y, Lu J, Chen Y, Jiang Q, Qin Q, Zhao L, Huang Q, Luo Z, et al. Long noncoding RNA LINC00511 contributes to breast cancer tumourigenesis and stemness by inducing the miR-185-3p/E2F1/Nanog axis. J Exp Clin Cancer Res CR. 2018;37:289.

17. Wang J, Tian $Y$, Zheng H, Ding Y, Wang X. An integrated analysis reveals the oncogenic function of IncRNA LINC00511 in human ovarian cancer. Cancer Med. 2019;8:3026-35.

18. Wang R-P, Jiang J, Jiang T, Wang Y, Chen L-X. Increased long noncoding RNA LINC00511 is correlated with poor prognosis and contributes to cell proliferation and metastasis by modulating miR-424 in hepatocellular carcinoma. Eur Rev Med Pharmacol Sci. 2019;23:3291-301.

19. Wang W, Lou W, Ding B, Yang B, Lu H, Kong Q, Fan W. A novel mRNAmiRNA-IncRNA competing endogenous RNA triple sub-network associated with prognosis of pancreatic cancer. Aging. 2019;11:2610-27.

20. Zhao X, Liu Y, Li Z, Zheng S, Wang Z, Li W, Bi Z, Li L, Jiang Y, Luo Y, et al. Linc00511 acts as a competing endogenous RNA to regulate VEGFA expression through sponging hsa-miR-29b-3p in pancreatic ductal adenocarcinoma. J Cell Mol Med. 2018;22:655-67. 
21. Li C, Liu H, Yang J, Yang J, Yang L, Wang Y, Yan Z, Sun Y, Sun X, Jiao B. Long noncoding RNA LINC00511 induced by SP1 accelerates the glioma progression through targeting miR-124-3p/CCND2 axis. J Cell Mol Med. 2019:23:4386-94.

22. Sun C-C, Li S-J, Li G, Hua R-X, Zhou X-H, Li D-J. Long Intergenic noncoding RNA 00511 acts as an oncogene in non-small-cell lung cancer by binding to EZH2 and suppressing p57. Mol Ther Nucleic Acids. 2016;5:e385.

23. Zhang H, Zhao B, Wang X, Zhang F, Yu W. LINC00511 knockdown enhances paclitaxel cytotoxicity in breast cancer via regulating miR-29c/CDK6 axis. Life Sci. 2019;228:135-44

24. Mao B-D, Xu P, Xu P, Zhong Y, Ding W-W, Meng Q-Z. LINC00511 knockdown prevents cervical cancer cell proliferation and reduces resistance to paclitaxel. J Biosci. 2019;44(2):44.

25. Yu C-L, Xu X-L, Yuan F. LINC00511 is associated with the malignant status and promotes cell proliferation and motility in cervical cancer. Biosci Rep. 2019;39(9):BSR20190903.

26. Zhang J, Sui S, Wu H, Zhang J, Zhang X, Xu S, Pang D. The transcriptional landscape of IncRNAs reveals the oncogenic function of LINC00511 in ERnegative breast cancer. Cell Death Dis. 2019;10:599.

27. Deng H, Huang C, Wang Y, Jiang H, Peng S, Zhao X. LINC00511 promotes the malignant phenotype of clear cell renal cell carcinoma by sponging microRNA-625 and thereby increasing cyclin D1 expression. Aging. 2019; 11(16):5975-91.

28. Liu L, Zhu Y, Liu A-M, Feng Y, Chen Y. Long noncoding RNA LINC00511 involves in breast cancer recurrence and radioresistance by regulating STXBP4 expression via miR-185. Eur Rev Med Pharmacol Sci. 2019;23:7457-68.

29. Moher D, Liberati A, Tetzlaff J, Altman DG. Preferred reporting items for systematic reviews and meta-analyses: the PRISMA statement. PLoS Med. 2009;6 Available from: https://www.ncbi.nlm.nih.gov/pmc/articles/PMC27075 99/. Cited 2019 Jun 20.

30. Stang A. Critical evaluation of the Newcastle-Ottawa scale for the assessment of the quality of nonrandomized studies in meta-analyses. Eur J Epidemiol. 2010;25:603-5.

31. Ottawa Hospital Research Institute. Available from: http://www.ohri.ca/ programs/clinical_epidemiology/oxford.asp. Cited 2019 May 31.

32. Tierney JF, Stewart LA, Ghersi D, Burdett S, Sydes MR. Practical methods for incorporating summary time-to-event data into meta-analysis. Trials. 2007;8:16.

33. Tang Z, Li C, Kang B, Gao G, Li C, Zhang Z. GEPIA: a web server for cancer and normal gene expression profiling and interactive analyses. Nucleic Acids Res. 2017:45:W98-102.

34. Tang Z, Kang B, Li C, Chen T, Zhang Z. GEPIAZ: an enhanced web server for large-scale expression profiling and interactive analysis. Nucleic Acids Res. 2019;47(W1):W556-60

35. Egger M, Smith GD, Phillips AN. Meta-analysis: principles and procedures. BMJ. 1997:315:1533-7.

36. Lee YH. An overview of meta-analysis for clinicians. Korean J Intern Med. 2018:33.277-83

37. Copas J, Shi JQ. Meta-analysis, funnel plots and sensitivity analysis. Biostat Oxf Engl. 2000;1:247-62.

38. Zhu F-Y, Zhang S-R, Wang L-H, Wu W-D, Zhao H. LINC00511 promotes the progression of non-small cell lung cancer through downregulating LATS2 and KLF2 by binding to EZH2 and LSD1. Eur Rev Med Pharmacol Sci. 2019; 23:8377-90.

39. Du X, Tu Y, Liu S, Zhao P, Bao Z, Li C, Li J, Pan M, Ji J. LINC00511 contributes to glioblastoma tumorigenesis and epithelial-mesenchymal transition via LINC00511/miR-524-5p/YB1/ZEB1 positive feedback loop. J Cell Mol Med. Available from: https://onlinelibrary.wiley.com/doi/abs/10.1111/jcmm.14829. Cited 2019 Dec 21

40. Liu F-T, Dong Q, Gao H, Zhu Z-M. The prognostic significance of UCA1 for predicting clinical outcome in patients with digestive system malignancies. Oncotarget. 2017;8:40620-32.

41. NCBI Resource Coordinators. Database resources of the National Center for Biotechnology Information. Nucleic Acids Res. 2018;46:D8-13.

42. Wang K, Zhu G, Bao S, Chen S. Long non-coding RNA LINC00511 mediates the effects of ESR1 on proliferation and invasion of ovarian cancer through miR-424-5p and miR-370-5p. Cancer Manag Res. 2019; Available from: https://www.dovepress.com/long-non-coding-rna-linc00511-mediates-theeffects-of-esr1-on-prolifer-peer-reviewed-fulltext-article-CMAR. Cited 2019 Dec 29
43. Chen Z, Wu H, Zhang Z, Li G, Liu B. LINC00511 accelerated the process of gastric cancer by targeting miR-625-5p/NFIX axis. Cancer Cell Int. 2019;19: 351.

44. Ding J, Yang C, Yang S. LINC00511 interacts with miR-765 and modulates tongue squamous cell carcinoma progression by targeting LAMC2. J Oral Pathol Med Off Publ Int Assoc Oral Pathol Am Acad Oral Pathol. 2018;47: 468-76.

45. Chen Y, Bao C, Zhang X, Lin X, Fu Y. Knockdown of LINC00511 promotes radiosensitivity of thyroid carcinoma cells via suppressing JAK2/STAT3 signaling pathway. Cancer Biol Ther. 2019;20:1249-57.

46. Li J, Li Y, Meng F, Fu L, Kong C. Knockdown of long non-coding RNA linc00511 suppresses proliferation and promotes apoptosis of bladder cancer cells via suppressing Wnt/ß-catenin signaling pathway. Biosci Rep. 2018;38(4):BSR20171701

47. Jiang L, Xie X, Ding F, Mei J, Bi R. Silencing LINC00511 inhibits cell proliferation, migration and EMT via PTEN/AKT/FOXO1 signaling pathway in lung cancer. Biochem Cell Biol. 2019. https://doi.org/10.1139/bcb-2018-0364.

\section{Publisher's Note}

Springer Nature remains neutral with regard to jurisdictional claims in published maps and institutional affiliations.
Ready to submit your research? Choose BMC and benefit from:

- fast, convenient online submission

- thorough peer review by experienced researchers in your field

- rapid publication on acceptance

- support for research data, including large and complex data types

- gold Open Access which fosters wider collaboration and increased citations

- maximum visibility for your research: over $100 \mathrm{M}$ website views per year

At BMC, research is always in progress.

Learn more biomedcentral.com/submissions 